\title{
Reliable Description of Preliminaries Item Using Civil Engineering Preliminaries Protocol (CEPP) In Conventional Contracts
}

\author{
Abdul Aziz Abas, Faridah Ismail, and Zulhabri Ismail $^{*}$, \\ Faculty of Architecture Planning and Surveying, Universiti Teknologi Mara, 40450 Shah Alam, Malaysia
}

\begin{abstract}
Civil engineering work deals with nature and thus, is exposed to enormous discrepancies due to nature's complexity compared to building works which are more certain. In the Malaysian construction industry, it is generally accepted that civil engineers administer civil engineering contracts and prepare tender documents. The Civil Engineering Preliminaries Protocol (CEPP) for conventional contracts is an ongoing research that deliberates on the cost-related items included in the Preliminaries. Preliminaries are subjective in nature and largely challenging to price. This paper considered previous research findings by conducting a literature review and accordingly, highlighted the problem statements raised on the contractual risks present due to the fallacy of item description. The identification of underlying problems and gaps within the area of study justifies the aim of the research to establishing a common protocol that is conversant to both engineers and contractors. The objective of the protocol is to eliminate disputes due to vagueness, ambiguities, and duplication of preliminary items in order to improve price accuracy. In practice, different approaches are taken by engineers and contractors in dealing with preliminary items. Engineers provide bills of preliminaries and contractors price them accordingly without establishing any mutual understanding and responsibility for risks. Conventional contracts prohibit contractors to provide their own preliminaries. Contractors instead have to obtain clarification on any ambiguities in the contract within the speculated time given during the tender period. As a way forward, the CEPP provides better clarity, accuracy, and transparency to engineers and contractors as well as the other construction players in general. Reliable descriptions of preliminary items ensure better price accuracy for the betterment of the construction industry.
\end{abstract}

\section{Introduction}

Civil engineering deals with the built environment and encompasses the design and construction of infrastructures such as bridges, dams and other civil works [1-3]. It also deals with nature and thus, is exposed to enormous discrepancies compared to building works which are more certain.

Preliminary items are subjective in terms of costs and largely challenging to price [4-9]. In Malaysia, the cost of preliminaries for civil work is between three to seven percent of the construction cost[10], but is unlikely to exceed $10 \%$ [11]. In the Malaysian construction industry, it is usual for civil engineers to administer civil engineering contracts [1-4]. It is generally accepted that engineers have to prepare tender documents [12]. Detailed descriptions of preliminary items is a crucial part of the tender to ensure correct pricing [13].

For conventional contracts, engineers are generally the originator of bills, such as bills for preliminary items; these bills are then handed to contractors to price [14] As such, contractors have no right to interfere during the

\footnotetext{
*Corresponding author: zulhabri@salam.uitm.edu.my
}

preparation of the document. Various approaches have been used by both engineers and contractors in preparing/construing the preliminary items list [10], [15]. Usually, engineers establish the bills of preliminaries for contractors to price accordingly, and take all the risks [7-9]. Nevertheless, contractors can obtain clarification for any ambiguity on the items listed within the tender period [19], [20]. This minimises misinterpretation that may lead to price errors.

\subsection{Preliminary items in Conventional Contracts}

The reliability of preliminary items has become a subject matter in the local construction industry and overseas due to its complexities [4], [6], [9], [11], [14-17]. The current procedure entails preliminaries to comprise of all important information, instructions and obligations, yet be descriptive in nature [17]. Preliminaries may also contain irrelevant items that may bring about higher bid prices [7]. 


\subsubsection{Derivation of Preliminaries}

Contracts are typically divided into five categories to meet different purposes, namely the traditional general contracts, design and build contracts, management contracts, hybrid contracts and miscellaneous contracts [24]. The traditional general contract is the only one that involves the Bills of Quantities which comprise of trade bills and preliminary items which form part of the contract [24].

The traditional general contract category correlates with the conventional contract and is commonly used in Malaysia since 1931 by the Public Works Department for construction projects [25]. In a conventional contract, preliminaries constitute a fraction of the documents but carry an important financial obligation to contractors alongside with trade bills [8], [26]. Preliminaries and trade bills are parts of the integrated Bills of Quantities (BQ) [3], [9-11]. Figure 1 shows an example of a preliminary that is generally included in conventional contracts.

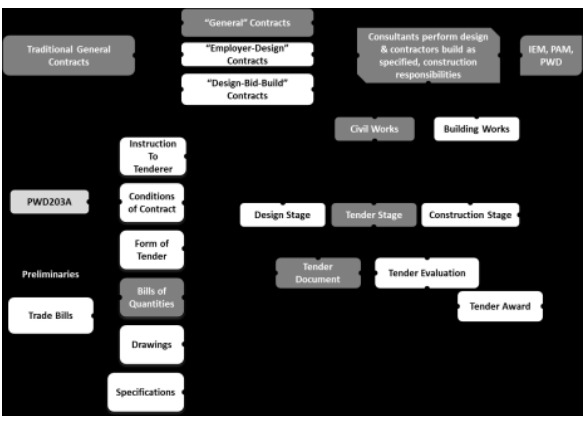

Fig. 1. Preliminaries in conventional contracts

\section{Literature Review}

\subsection{Underlying Issues of Preliminaries}

In conventional civil engineering contracts, it is prerogative that the engineer establishes the preliminary items and trade bills to constitute the Bills of Quantities (BQ) [6], [17], [27]. Engineers have used various means ranging from bespoke methods to more organised means. During the tender stage, contractors use their own interpretation and judgement to price the items [28], [29] irrespective of the items' reliability. Preliminary items are the most difficult to price as its pricing is arbitrary [29]. Contractually, contractors take full responsibility of the bills although they are not the ones pricing them.

The approaches taken by the two exponents are distinct, and there is no common platform that can be used to provide a mutual understanding between engineers and contractors; thus, causing huge price variations [16], [17], [21]. A protocol, namely the Civil Engineering Preliminaries Protocol (CEPP) has been established to promote the reliability of preliminary items' descriptions in order to avoid disputes and establish better price accuracy. The description of items has garnered a lot of attention due to its susceptibility to discrepancies, repetitiveness, insufficient deliberation, manipulation, arbitration and most of all, to be definitive [7], [29-31].

\subsubsection{Various Methodologies Used by Engineers}

Various methodologies have been adopted by engineers to establish the preliminary items that form a part of the BQ. Due to the complexity and uncertainty of civil works, engineers would usually adopt the descriptions of preliminary items from past projects [28] with modification in order to generate the general bills of a project based on their best assumptions and experiences.

Engineers do exploit the descriptions of preliminary items from past projects for new projects [7]. This is easily understood as an easier way to conclude the description of cost-related preliminary items. This is typically done due to either insufficient preparation time given to prepare the tender documents, or having a past project of a similar nature. This action, if not cautiously measured for necessary adjustment to suit a project's needs, may lead to discrepancies in the items' description [31]. Lack of information may also lead to the omission of important items which thus may incur additional contract costs due to additional orders [2729].

Engineers expect contractors to be meticulous in their pricing of preliminaries, and to price all included items [6], [16], [35]. However, a problem exists in most cases where the description of items is not adequately deliberated on in order to specify the requirement [7]. Though contractors are required to raise queries pertaining to incomplete or ambiguous information before the closing of a tender, certain contractors opt for passive action and prefer to be silent in hope that the issue(s) raised will be resolved in time [7]. The writer opines that it is the engineers' responsibility to provide a reliable and clear description of the preliminary items, particularly for cost-related items. Figure 2 depicts the present common approach taken by engineers to conclude preliminaries.

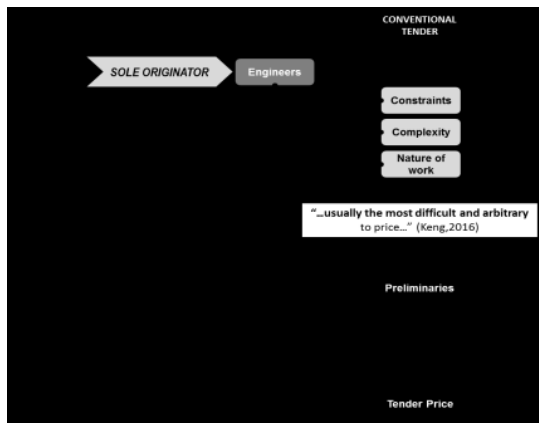

Fig. 2. Typical approach by engineers to establish BQ 
There are several formats used in the present practice adopted by engineers ranging from the simplest to the most tedious forms of preliminaries [15]. The writer suggests the usage of a codified format by means of a common protocol to minimise discrepancies and promote better transparency.

\subsubsection{Various Approaches by Contractors to Construe Preliminaries}

Contractors are obliged to price preliminaries in compliance with a tender's requirements within a specific tender period. During the process, various approaches are taken to construe and anticipate the workload, timespan, direct and indirect costs, and requirements for suppliers or sub-contractors. Contractors use the pretext of a project's complexities to react accordingly to the bills of preliminaries. Site information obtained during site reconnaissance which is either organised by the employer or on the contractor's own initiative is important [32].

Contractors predominantly price cost-related preliminaries based on their own ideas and anticipation [28], [29]. Nonetheless, they depend entirely on the reliability of item description as prepared by the engineers. Despite their experience, contractors usually have systematic approaches to this process such as anticipating all possible site constraints, pricing strategies with the latest price statistics and their own experience in a similar project environment, striking good deals with local labours and suppliers, and working with experienced managers [32]. Figure 3 depict contractors as the sole receiver who complies with a tender or contract's requirements based on their own interpretations.

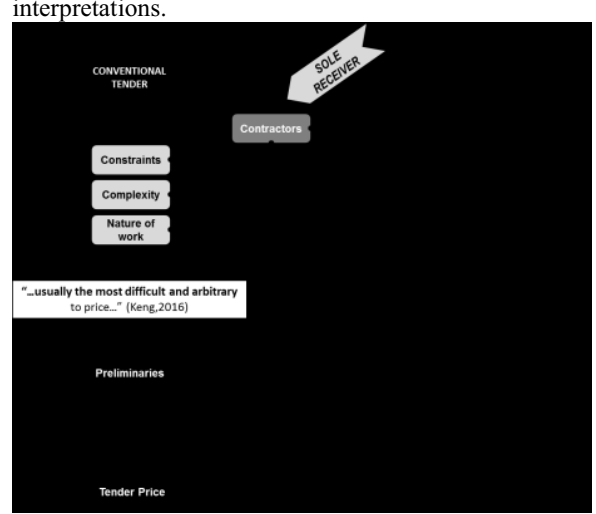

Fig. 3. Typical approach by contractors in interpreting preliminaries

Contractors are expected to price all items included in the preliminaries despite the complexities and uncertainties [16]. The contractors in return take drastic actions to minimise their risks by jacking up the prices of other items [7], or instead accept the risks by charging a lower price with a reduced profit margin due to desperation to secure a contract [32].
Contractors seldom take proactive actions when dealing with incomplete information. They normally stay silent and expect the issue to resolve itself over time [7]. Contractors simply comply with the term of tender or "compliance to the bid" during the tender exercise to avoid unnecessary troubles that may reduce their chances of winning the contract bid.

\section{Research Methodologies}

A literature review was carried out on 41 research or academic papers from multi-platforms not limited to Scopus, Google Scholar, conferences, symposiums, and online journals. References to two online newspapers for issues not available from previous research were also carried out. A qualitative analysis approach was adopted. The protuberant issues were discussed to arrive at a coherent explanation. Problem statements were generalised with emphasis on the attitudes of engineers and contractors. The objective is to find the imperative criteria necessary to institute a reliable description of preliminary items. The findings set forth a way forward for a common protocol conversant to the construction proponents.

\section{Findings and Discussions}

Items of preliminaries are arbitrary and thus, are difficult to be given accurate price justification [29]. The writer agrees that as preliminaries tend to contain all particulars namely general information, contractual items, specific project or client's requirements, temporary work, and reminders about the obligation of the contractors, it is therefore common that the preliminary item form contains general information, general instructions and recurring items intermingled with each other. Unrelated information unnecessary to the course of the project are also usually incorporated [7]. Such unrelated items need to be discarded. The approach needs to be simplified for easy understanding, thus making the items more definitive in description.

The present approach to conventional contracts does not require early contractor involvement (ECI) during the preparation of the tender documentation. Contractors are only required to submit the price bid during the tender stage. Instead of the present approach, contractors should be invited to participate at the early stage in order to provide construction advice and early identification of risks [36]. This would be a theoretical approach that can be adopted to improve the quality and reliability of preliminary items' descriptions. This approach may be advantageous for medium and large projects in terms of the development costs involved. However, its implementation for small scale projects may not be feasible.

The measurement of cost-related preliminary items is a serious challenge as it should be codified and made familiar to both contractors and engineers [7]. The terminologies, construction techniques, descriptions of items and measurement approaches used by the engineers must be clear, transparent and precise. All 
matters included in the preliminaries must be definitive in order to provide considerable understanding for the contractors to price [30].

The need for a common protocol is prerogative to bring together the engineers and contractors for mutual understanding of what the other does and anticipates. Figure 4 emphasises the importance of a common protocol to be adopted in order to establish reliable preliminaries which will eventually lead to a reliable tender price.

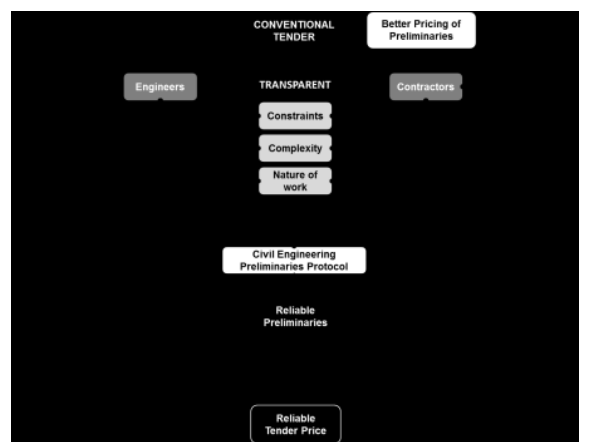

Fig. 4. Common Protocol Influencing Reliability of Preliminaries and Tender Price

Consideration should be given to ensure all vague or indeterminate areas are sufficiently clarified, and due effort should be given to exclude uncertainties from the tender documentation [37]. The writer opines that engineers should meticulously conduct the due diligence study and acquire sufficient practical experience of similar nature of work in order to be able to prepare precise preliminary item forms. Another important facto that should be considered is coordination between the description of preliminary items and the trade bills in order to avoid duplication or undue conflicts or omissions [38]

Different approaches and understandings to the preliminary items between engineers and contractors often cause unnecessary disputes [4]. The writer anticipates that disputes may occur if the description of preliminary items is vague or/and contractors purposely manipulate the price of specific items for their own advantage. It becomes worse if the manipulation is done by the employer's party, namely the engineers and is complicated by misunderstandings on the side of the contractors [33].

It is common practice in Malaysia and other countries that cost-related preliminary items are measured entirely in lump sum [20], [21], [39] with long-winded descriptions. Unfortunately, the breakdown of items is not mandatory, thus the components of the lump sum price are not verified. For such circumstances, contractors provide unverified or arbitrary lump sum figures on the preferred items based on their own interpretation and anticipation [16], [29]. However, through a more drastic approach, accurate and detailed information on the itemised preliminaries may provide better results and make the items easier to price [7]. The breakdown of items is therefore important to the attainment of the objective [23], [35], [40].

Indirect costs e.g. off record 'compensation' or 'protection money' [41], [42] and on-site authority requirements are usually the main contributors related to the cost of preliminaries during construction. Despite being important, contractors are unable to ascertain the extent of its impact at the point of tender, thus are seldom considered as an integral part of the contract. Though intangible in nature, indirect costs cannot be neglected as a critical cost component associated with the construction process [43]. The preliminary items for indirect costs can be used to cushion some unexpected cost impacts.

\section{Conclusions}

It is construed that engineers and contractors have differences in establishing/construing the preliminary items. The description of preliminary items must be definitive in order to provide considerable understanding for contractors to price, hence promote better price accuracy. Reliable descriptions of preliminary items that are easy to comprehend lead to reliable tender prices.

As a way forward, a common understanding between the parties in particular and other construction proponents in general through a codified protocol is deemed important. The Civil Engineering Preliminaries Protocol (CEPP) is an on-going research that leverages on the approaches of both engineers and contractors. Differences are mediated by studying the breakdown of items, unit of measurements, use of terminologies, construction techniques, and definitive descriptions of each item. The modification and introduction of new elements such as provisional sum, unexpected indirect costs such as compensation, Early Contractor Involvement (ECI) and Early Stakeholder Involvement (ESI) are among the main elements given attention.

The criteria for reliable description of preliminary items pragmatically include but are not limited to; (1) cautious adoption from past projects, (2) relevance, (3) definitiveness, (4) easy comprehension, (5) elimination of discrepancies, repetitiveness, insufficient deliberation, manipulation, and arbitration, (6) clear, transparent and precise description, (7) avoidance of uncertainty, (8) coordination with trade bills to avoid undue problems, (9) breakdown of items to provide clarity and accuracy, (10) indirect costs as a critical cost component, and (11) measurement of cost-related preliminary items codified and familiar to contractors and engineers.

In a nutshell, the CEPP should contain the above criteria to provide better clarity, accuracy, and transparency to engineers and contractors as well as the other construction proponents in general. Reliable description of preliminaries item ensures better price accuracy for the betterment of the construction industry. 


\section{References}

1. J. Lucas, What is civil engineering?, Live Science, (2014)

2. Columbia University, What is civil eng.?, (2016)

3. S. M. Vera, Diff. Between Arch., Civil Eng And Bldg Eng World Wide, (2013)

4. R. A. Jimoh and S. M. Adama, Assessment of prelim. in relation to the total cost of renovation work in public schools in Abuja, Nigeria, in ICEC IX World Congress Assessment, 1-9, (2011)

5. H. S. Kammer, Pricing Prelim., (2012)

6. A. A. Abas, Z. Ismail, F. Ismail, and R. A. Arshad, Bills of Prelim. Pricing Trends for Malaysian Civil Infra. Works in Conventional Contract, (2017)

7. H. Adnan and A. Nawawi, Bills of Quantities: Perspectives of Contractor in Malaysia., J. Appl. ..., 5, 863-873, (2011)

8. T. S. Yi, A study Into prelim. for the constr. project in Setapak, Wangsa Maju, Kuala Lumpur, (2010)

9. H. Gebreab, 'Tender Cost Preparation', Academia 11, (2016)

10. A. A. Abas, Z. Ismail, F. Ismail, and R. A. Arshad, Cost of Prelim. of Constr. Projects for Civil Infra. Works, Sains Humanika, 9, 9-13, (2017)

11. Turner \& Townsend, Int. const. market survey 2016, (2016)

12. K. H. Chan, Shortage of Engrs. and What That Means in Const. Disputes, Kheng Hoe Advocates, (2016)

13. K. A. EL Nahel, What Are Prelim. in Const. Contracts? How to Use Prelim. Costs in Claims. (2014)

14. A. A. U. S. A. U. S. Gunathilaka and L. D. I. D. I P. Senevirathne, Common Errors That Are Being Made In Preparing And Pricing BOO In Sri Lanka Const. Industry, in The second world construction symposium 2013: Socio-economic sustainability in construction, 20021, 93-100, (2013)

15. T. Cunningham, The Function and Format of $B Q$ : an Irish Context, (2016)

16. N. A. Ghani, The importance of prelim. items, (2006)

17. A. A. Abas, R. A. Arshad, and Z. Ismail, Challenges of Accurate Estimation in Prelim. of Constr. Projects, Adv. Sci. Lett., 23, 29702973(4), (2017)

19. K. Potts, Construction Cost Management: Learning from Case Studies, 28, (2008)

20. H. Deen, The Lump Sum Const. Contract - What Seems to be the problem, (2011)

21. A. A. Abas, Variation in Price of Prelim. in Constr. Projects During Tender Stage, (2018)

23. S. O. Oyediran, O. M. Ajayi, and F. A. Aderinto, Cost Modeling of Prelim. for Bldg. Projects, Built Environ. J., 9, 1-8, (2012)

24. S. K. S. Harbans, Const. Contracts: An Overview, Ing., 6-20, (2005)

25. S. Rajoo, Standard Forms of Contract - The Malaysian Position, in Int. Bar Assoc. (IBA) Annual Conference 2014, (2014)

26. F. Aderinto, Admin. of project prelim.
Construction Week Online.com, Feb-2010

27. The Entrusty Group, What are Prelim. and How to Evaluate Them?, Master Build. J., 1, 80-90, (2009)

28. A. C. Haruna, A. Mohammed, and G. S.-P. Buba, Appraising Contractors' Perception of Pricing Prelim. of Housing Projects in Kaduna, Nigeria, FUW Trends Sci. Technol. J., 2, 1008-1012, (2017)

29. T. C. Keng, Costing for The Prelim. Section of The Constr., in Cost and Contract Administration in Construction, Devine Perspectives, 24-29, (2016)

30. S. Bandi, Bills of Quantities: Raison d 'etre? Academia, 2, 1-15, (2016)

31. L. I. Yanqiu and K. E. Hong, Research on Adjustment of Prelim. Caused by New Work Sections And Trades added in list, in 2nd Int. Conference on Education Technology, Management and Humanities Science (ETMHS 2016) Research, 170-174, (2016)

32. C. Ji, J. Mbachu, and N. Domingo, Factors Influencing the Accuracy of Pre-Contract Stage Estimation of Final Contract Price in New Zealand, Int. J. Constr. Supply Chain Manag., 4 51-64, (2014)

33. T. Cunningham, Contractors 'Claims for Loss and Expense under the Principle "Traditional "Forms of Irish Building Contract, 31, (2014)

35. T. Cunningham, ARM4 - A Working Guide Section B - Preliminaries, 0-18 (2015)

36. M. Frazer, Australia: Early contractor involvement in preliminary design process of constr. projects, Mondaq Ltd., (2012)

37. Trevorsadd, Prelim. (General Site Costs) A Guide to the Pricing and Use of Prelim. in the Formulation of Budgets, Quotations and Tenders, 16, (2005)

38. S. Doran, Preliminaries and contemporary procurement, The NBS.com, (2007)

39. R. C. Smith, Estimating and Tendering for Building Work, (2013)

40. NJDOT, Cost Estimating Guideline, Progr. Manag. Off., 126, (2016)

41. H. S. Shahrudin, 4 men arrested for attempting to extort RM50,000 from contractor, New Strait Times, 26-Oct-2017

42. E. S. M. Chin, Gangsters on mean streets, Malay Mail Online, Aug-2016

43. R. K. Ellsworth, Estimating indirect costs for infra. constr. projects, Construction Accounting \& Taxation, 21, 11-16 (2011) 